\title{
Non-Menstrual Toxic Shock Syndrome Following Breast Abscess - A Rare Case Report From Nepal
}

\author{
Aabishkar Bhattarai, ${ }^{1}$ Deependra Gurung, ${ }^{2}$ Bijaya Karki ${ }^{3}$ \\ ${ }^{1}$ Manipal College of Medical Sciences, Pokhara, ${ }^{2}$ Department of Emergency Medicine, Gandaki Medical College Teaching \\ Hospital Pokhara, ${ }^{3}$ Department of Intensive Care Unit, Biratnagar Aspatal Pvt. Ltd. Birat Nursing Hone, Biratnagar, Morang, \\ Nepal.
}

\begin{abstract}
Introduction

Toxic Shock Syndrome is an acute illness caused by exotoxin producing Staphylococcus or Group A Streptococcus. TSS was traditionally associated with high absorbency tampon use in menstruating women until eventually, these were taken off the market. Since then, non-menstrual causes including wound infections, abscess, mastitis, burns, osteomyelitis, retained foreign bodies, etc are leading causes of TSS. TSS is characterized by fever, headache, myalgia, abdominal pain, hypotension, non-blanchable ery thematous rash progressing to multi-organ damage. The mainstay of treatment includes removal of source of infection, adequate volume resuscitation, vasopressors and antibiotic coverage. Despite adequate treatment, the case fatality rate of Streptococcal TSS is $50 \%$ and Staphylococcal TSS is $3 \%$. Here we present a unique case of 24 -year-old women who presented with fever, headache, abdominal pain and hypotension 6 days after self-drainage and self-medication of breast abscess. She was diagnosed with TSS secondary to breast abscess and admitted to Intensive Care Unit (ICU), resuscitated and successfully treated with antibiotics.
\end{abstract}

Keywords: breast abscess; Staphylococcus aureus; Toxic Shock Syndrome.

\section{INTRODUCTION}

The most common organism causing breast abscess is Staphylococcus aureus. The most common organisms leading to Toxic Shock syndrome (TSS)are virulent strain of Staphylococcus aureus and Streptococcus pyogens.TSS is mostly associated with high absorbency tampon use in menstruating females but is also associated with non- menstrualcauses including surgical and postpartum wound infections, mastitis, septorhinoplasty, sinusitis, osteomyelitis, arthritis, burns etc. ${ }^{1}$ Toxic shock syndrome is rarely reported in breast abscess without accompanying mastitis. There is no known case of Toxic Shock Syndrome published till date from Nepal. The overall incidence of TSS varies from 0.8 -3.4 per 100,000 population in USA. ${ }^{2}$ The incidence in Nepal

Correspondence: Dr. Aabishkar Bhattarai, Manipal College Of Medical Sciences, Pokhara, Nepal. Email: aabishkar. bhattarai@gmail.com. Phone: +977-9846077855. 
may be much lower due to very limited use of tampons by Nepalese women. The case fatality rate of Streptococcal TSS may exceed $50 \%$, particularly with delayed diagnosis; whereas Staphylococcal TSS is less than $3 \% .^{3}$ Recurrences are common among women who continue to use tampons during the first 4 months after an episode of TSS.

\section{CASE REPORT}

24-year-old female came to the Emergency Department with history of fever for 3 days. Fever was acute, intermittent, associated with chills, rigors and sweating with maximum temperature of 102 F. She also reported headache, generalized body weakness, diffuse muscle pain, non-localizing diffuse abdomen pain and reduced urine output. 6 days prior she attempted self-drainage of 2-3 $\mathrm{ml}$ pus from a small pustule in her right breast and self-medicated herself with Amoxicillinclavulanate and analgesics. At Emergency Department she was oriented to time, place and person but was ill looking and irritable. Blood pressure was $80 / 60 \mathrm{~mm}$ of $\mathrm{Hg}$, Pulse $110 / \mathrm{min}$, Respiratory rate of 16 cycles/min and oxygen saturation of $90 \%$ in room air. Abdominal examination reveals non tender abdomen without guarding and rigidity. Respiratory and cardiovascular examination were normal. There were no signs of meningeal irritation and focal neurological deficits. Breast examination revealed mild tenderness over the right upper quadrant with approximately $1 \mathrm{~cm}$ of tender mass accompanied with pus point without skin erythema or cellulitis.

Laboratory investigations revealed following: WBCs: $16,000 / \mathrm{mm}^{3}$ with $82 \%$ neutrophils;

PT/INR/Aptt: 17/1.2/38.8

Urea:13; Creatinine:0.6; Sodium:142 meq/l; Potassium:3.7 meq/1
Urine Analysis:16-18 pus cells/hpf

AST:70 U/L; ALT:90 U/L; Serum bilirubin(total):0.7 mg/dl

Chest Xray and Electrocardiography were normal.

Ultrasonography of right breast revealed small pus-filled area with 2-3 ml fluid in the right upper quadrant at 10-12 o' clock position. Surgical drainage of the breast abscess was performed in ER.

Widal test (for Salmonella typhi and Para typhi) and Tropical fever comprehensive panel including Dengue, Leptospira, Rickettsia, West Nile, Plasmodium and Chicken Guinea were negative. Urine and Pus were sent for Gram stain and Culture/Sensitivity and patient was admitted in Intensive Care Unit and managed with fluid resuscitation, intravenous vasopressor (Nor-adrenaline via central line) and antibiotics (Injection PiperacillinTazobactam and Injection Doxycycline).

On second day of ICU admission, she developed blanchable erythematous rashes all over the body. On third day, urine culture revealed sterile growth; pus culture revealed Methicillin Resistant Staphylococcus aureus sensitive to piperacillin-tazobactam and vancomycin. Patient was started with Inj. Vancomycin and Inj. Clindamycin on $3^{\text {rd }}$ day. On $5^{\text {th }}$ day of admission, she developed peeling of the skin from palms and soles (Figure 1). Blood pressure was maintained with tapering dose of nor-adrenaline which was stopped in $5^{\text {th }}$ day of ICU admission. The patient improved over 1 week and was discharged after a 7-day course of Injection Vancomycin and Clindamycin. 


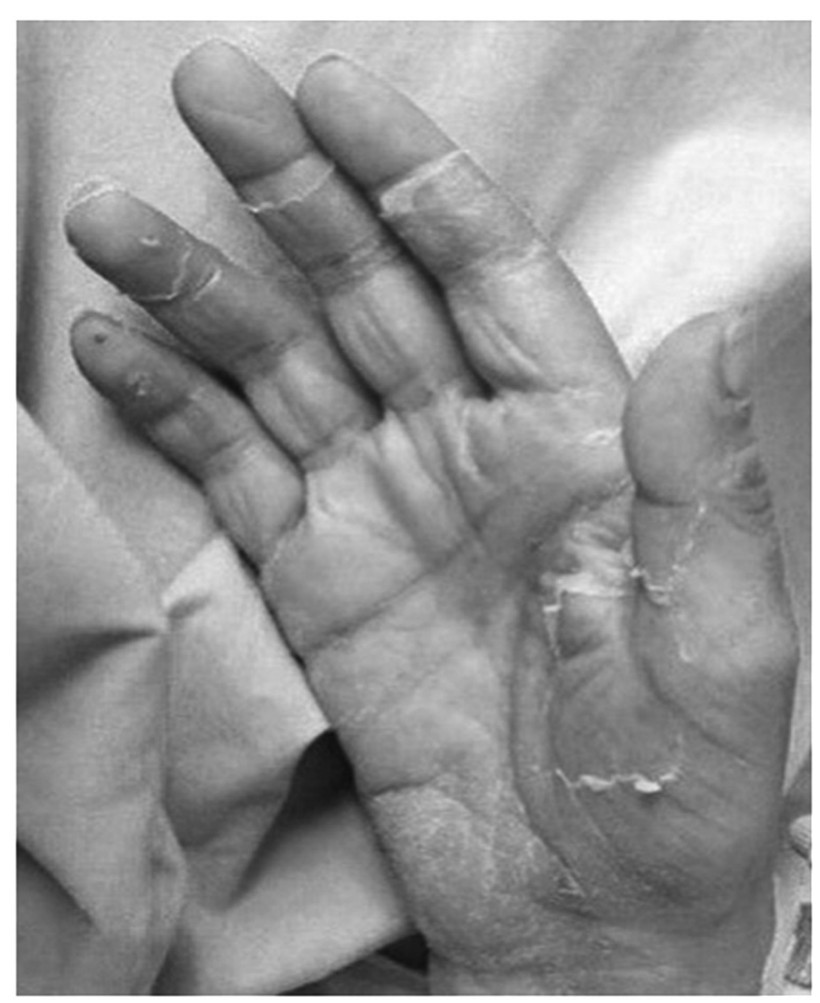

Figure 1. Skin peeling from the palm.

\section{DISCUSSION}

Staphylococcus aureus produce exotoxins that leads to food poisoning, caused by ingestion of $S$. aureus enterotoxin; scalded skin syndrome, caused by exfoliative toxin; and toxic shock syndrome (TSS), caused by toxic shock syndrome toxin-1 (TSST1). S. aureus TSST-1exotoxins are superantigens which are able to activate large numbers of $\mathrm{T}$ lymphocytes, resulting in massive cytokine production. Massive release of interleukin (IL)-1, IL-2, tumor necrosis factor (TNF)-alpha and TNFbeta, and interferon (IFN)-gamma from $\mathrm{T}$ cells, results in the signs and symptoms of TSS. ${ }^{4}$

Women having staphylococcal colonization of the vagina and who use tampons or other devices (e.g., contraceptive sponges, diaphragms) for prolonged period in the vagina are at high risk ofacquiring Toxic shock syndrome. ${ }^{5}$ However, TSS can also present in non-menstrualsettings such as in soft tissue infections, post-surgical infections, burns, retained foreign bodies such as nasal packing, and dialysis catheters. Staphylococcal TSS typically results from localized infection such as an abscess, whereas streptococcal TSS arises from systemicbacteraemia, necrotizing fasciitis, or cellulitis. ${ }^{1}$

The clinical features of TSS develops rapidly with fever, chills, malaise, headache, sore throat, fatigue, vomiting, diarrhoea and abdomen pain progressing to hypotension, generalised body rash and multi-organ failure. Initially the rash is macular, predominantly on the chestbut later progresses to a diffuse, blanching, erythroderma. The rash desquamates one to two weeks later followed by full-thickness skin peeling. ${ }^{2}$

According to CDC, the diagnosis criteria for Staphylococcal TSS includes:

Hypotension (SBP $<=90 \mathrm{~mm}$ of $\mathrm{Hg}$ for adults or $<5^{\text {th }}$ percentile by age for children $<16$ years)

Multi-organ involvement $(>=3$ of the following):

- Gastrointestinal (vomiting or diarrhoea at onset of illness)

- Muscular (severe myalgias or increased creatinine phosphokinase $2 \times$ normal)

- Mucous membranes (oropharyngeal, conjunctival or vaginal hyperaemia)

- Renal (BUN and creatinine elevation $>2 x$ normal, presence of pyuria i.e $\mathrm{WBC}>=5 /$ hpf)

- Hepatic (Transaminase or bilirubin at least $2 \times$ the upper limit of normal)

- Hematologic (platelets $<100,000 /$ microliter)

- Central nervous system (altered mental status, disorientation, without focal neurologic deficits)

A patient with fever $>38.9^{\circ} \mathrm{C}$, hypotension, diffuse erythroderma, desquamation and involvement of at least three organ systems is a confirmed case of Staphylococcal TSS. A probable case, is a patient who is missing one 
of the characteristics of the confirmed case definition.

Streptococcal TSS presents with additional symptoms at the site of infection. Patients may present with severe pain at the site of the softtissue infection with associated necrotizing fasciitis, myositis or gangrene. Respiratory system involvement can occur leading to acute respiratory distress syndrome in about $55 \%$ of patients. $^{5}$

The mainstay of treatment for TSS is supportive. A meticulous search for the presence of foreign material in the vaginal canal (e.g., vaginal tampons, douches, contraceptive sponges,) or nasal packings should be done and these materials should be removed. Drainage of any identified infectious focus (e.g. breast abscess) or exploration of surgical wounds is mandatory.

Fluids and electrolytes are replaced to prevent or treat hypovolemia, hypotension, and shock. Because fluid loss occurs into third compartment of the body (because of systemic capillary leakage and hypoalbuminemia), there may be profound shock resistant to volume resuscitation alone. Aggressive fluid resuscitation and circulatory, ventilatory, and/or haemodialysis support are sometimes required. Obvious infections should be treated with antibiotics. Until the culture results are available, empiric treatment with Clindamycin and Vancomycin is done. If a pathogen is isolated in culture, the antibiotic regimen is adjusted as below:

- For group A streptococci: Clindamycin plus a Beta-lactam

- For methicillin-susceptible S.

- ureus (MSSA): Clindamycin plus Oxacillin or Nafcillin

- For methicillin-resistant Staphylococcus aureus (MRSA): Clindamycin plus
Vancomycin or Linezolid alone or in combination with Vancomycin.

Antibiotics given during the acute illness may eradicate pathogen foci and prevent recurrences. Passive immunization to TSS toxins with Intravenous immune globulin (IVIg) has been helpful in severe cases of both types of TSS especially in patients that are recognized early in their course who have not responded to fluid resuscitation and vasopressors. There is no role of corticosteroid in treatment of Toxic shock syndrome. The disease may not induce active immunity, so recurrences are possible. ${ }^{5}$

\section{CONCLUSION}

Toxic shock syndrome is a life-threatening complication of Group A Streptococcus or Staphylococcus aureus infection. Most cases are secondary to tampon use during menstruation however non menstrual cases are increasing these days due to removal of highly absorbable tampons from the market and patient education. Breast abscess leading to TSS is rarely mentioned in literature. We presented a case of young women with selfdrainage of breast abscess who presented to ER with fever, myalgia, headache, abdomen pain, altered mental status and hypotension and was treated with abscess drainage, fluid and vasopressor resuscitation, and antibiotics in Intensive Care Unit. As per our knowledge, this is a first reported case of non-menstrual TSS from breast abscess in Nepal.

Conflicts of interests: No conflicts of interest occur among the authors.

Funding: No fund was provided for this study. 


\section{REFERENCES}

1. Vincent JL, Mongkolpun W. Current management of Gram-negative septic shock [Internet]. Vol. 31, Current opinion in infectious diseases. NLM (Medline); 2018 [cited 2020 Jul 6]. p. 600-5. Available from: https://pubmed.ncbi.nlm.nih. gov/30299358/

2. Toxic Shock Syndrome - StatPearls - NCBI Bookshelf [Internet]. [cited 2020 Jul 4]. Available from: https://www.ncbi.nlm. nih.gov/books/NBK459345/

3. Schmitz M, Roux X, Huttner B, Pugin J. Streptococcal toxic shock syndrome in the intensive care unit. Ann Intensive Care [Internet]. 2018;8(1):1-10. Available from: https://doi.org/10.1186/s13613-018-0438-y

4. Schlievert PM, Jablonski LM, Roggiani M, Sadler I, Callantine S, Mitchell DT, et al. Pyrogenic toxin superantigen site specificity in toxic shock syndrome and food poisoning in animals. Infect Immun [Internet]. 2000 Jun [cited 2020 Jul 6];68(6):3630-4. Available from: /pmc/ articles/PMC97652/?report=abstract

5. Toxic Shock Syndrome (TSS) Infectious Diseases - Merck Manuals Professional Edition [Internet]. [cited 2020 Jul 6]. Available from: https:// www.merckmanuals.com/professional/ infectious-diseases/gram-positive-cocci/ toxic-shock-syndrome-tss

6. Toxic Shock Syndrome (Other Than Streptococcal) | 2011 Case Definition [Internet]. [cited $2020 \mathrm{Jul}$ 4]. Available from: https://wwwn.cdc.gov/nndss/ conditions/toxic-shock-syndrome-otherthan-streptococcal/case-definition/2011/

Citation: Bhattarai A, Gurung D, Karki B. Non-Menstrual Toxic Shock Syndrome Following Breast Abscess - A Rare Case Report From Nepal. JCMS Nepal. 2021 ; 17(1); 88-92. 